\title{
Rigidity Results for Elliptic PDEs with Uniform Limits: \\ an Abstract Framework with Applications
}

AlbERTO FARINA \& ENRICO VALDINOCI

\begin{abstract}
We provide an abstract framework for a symmetry result arising in a conjecture of G.W. Gibbons and we apply it to the fractional Laplace operator, to the elliptic operators with constant coefficients, to the quasilinear operators, and to elliptic fully nonlinear operators with possible gradient dependence.
\end{abstract}

\section{INTRODUCTION}

Let $u: \mathbb{R}^{n} \rightarrow \mathbb{R}$ be a solution of the problem

$$
\begin{cases}\operatorname{Lu}(x)=f(u(x)) & \text { for any } x \in \mathbb{R}^{n}, \\ \lim _{x_{n} \rightarrow \pm \infty} u\left(x^{\prime}, x_{n}\right)= \pm 1, & \text { uniformly in } x^{\prime} \in \mathbb{R}^{n-1}\end{cases}
$$

Here, $L$ is an operator (not necessarily linear) acting on a space $\mathbb{X}$ of smooth (say, $C^{r}$ with $r \geqslant 1$ ) functions and commuting with the translations, i.e.,

$$
L(u(x+y))=(L u)(x+y) \quad \text { for any } y \in \mathbb{R}^{n},
$$

whose precise assumptions will be listed below.

The space $\mathbb{X}$ is supposed to contain functions from $\mathbb{R}^{n}$ to $\mathbb{R}$ and to be translation invariant (with respect to the translations in $\mathbb{R}^{n}$ ), that is

(1.3) if $u \in \mathbb{X}$, then the functions $x \mapsto u(x+y)$ lie in $\mathbb{X}$ too, for any $y \in \mathbb{R}^{n}$.

We remark that we need that the space $\mathbb{X}$ is translation invariant, as in (1.3), because we want to study the operator on both the function and on its translation. 
On the other hand, we do not need that the operator itself is translation invariant, but only that it commutes with the translations, as in (1.2). This technical detail will allow us to deal with nonlinear operators too.

As for the nonlinearity, we suppose that $f \in C^{1}(\mathbb{R})$, with

$$
\inf _{r \in(-\infty,-1] \cup[1,+\infty)} f^{\prime}(r)>0 .
$$

A paradigmatic example of nonlinearity satisfying the above assumptions is the function $f(r)=r^{3}-r$.

The goal of this paper is to prove that $u$ possesses one-dimensional symmetry, that is, that there exists $u_{0}: \mathbb{R} \rightarrow \mathbb{R}$ such that

$$
u\left(x^{\prime}, x_{n}\right)=u_{0}\left(x_{n}\right) \quad \text { for any }\left(x^{\prime}, x_{n}\right) \in \mathbb{R}^{n-1} \times \mathbb{R} .
$$

For this, the following hypotheses are taken on $L$ :

H1. (Linearization): If $\varphi \in \mathbb{X}$ satisfies $L \varphi=f(\varphi)$ in $\mathbb{R}^{n}$, then there exists an operator $\tilde{L}$ acting on some space of functions $\tilde{\mathbb{X}}$, with $\tilde{\mathbb{X}}$ translation invariant in the sense of (1.3), such that $\partial_{\omega} \varphi \in \mathbb{\mathbb { X }}$ for any $\omega \in S^{n-1}$ (where, as usual, $\partial_{\omega}$ denotes the directional derivative) and

$$
\tilde{L}\left(\partial_{\omega} \varphi\right)=f^{\prime}(\varphi) \partial_{\omega} \varphi \quad \text { in } \mathbb{R}^{n} .
$$

H2. (Compactness): If $\varphi \in \mathbb{X}$ satisfies (1.1), $x^{(k)} \in \mathbb{R}^{n}$ and $\varphi^{(k)}(x):=$ $\varphi\left(x+x^{(k)}\right)$, we have that there exists a function $\varphi^{(\infty)} \in \mathbb{X}$ such that, up to a subsequence,

$$
\begin{aligned}
& \lim _{k \rightarrow+\infty} \varphi^{(k)}(x)=\varphi^{(\infty)}(x), \\
& \lim _{k \rightarrow+\infty} \nabla \varphi^{(k)}(x)=\nabla \varphi^{(\infty)}(x), \\
& \lim _{k \rightarrow+\infty} L \varphi^{(k)}=L \varphi^{(\infty)},
\end{aligned}
$$

for any $x \in \mathbb{R}^{n}$.

H3. (Maximum Principle for the linearized operator): If $w \in \widetilde{X}$ satisfies $\tilde{L} w=c(x) w$ in $\mathbb{R}^{n}$, with

$$
w(x) \geqslant 0 \text { if }\left|x_{n}\right| \leqslant M \text { and } c(x) \geqslant \kappa \text { if }\left|x_{n}\right| \geqslant M,
$$

for some $\kappa>0$ and $M>0$, then

$$
w(x) \geqslant 0 \quad \text { for any } x \in \mathbb{R}^{n} .
$$

H4. (Strong Maximum Principle for the linearized equation): If $v \in \tilde{\mathbb{X}}$ satisfies $\tilde{L} v=f^{\prime}(\varphi) v$, for some $\varphi \in \mathbb{X}$, and $v \geqslant 0$ in $\mathbb{R}^{n}$ with $v(0)=0$, then $v$ vanishes identically. 
H5. (Maximum Principle for the difference operator): Given $\varphi \in \mathbb{X}$, let

$$
\underline{L}_{\varphi} w(x):=L(\varphi+w)(x)-L \varphi(x) .
$$

Let $U$ be an open set contained in $\left\{x_{n} \leqslant \mu_{-}\right\} \cup\left\{x_{n} \geqslant \mu_{+}\right\}$, for some $\mu_{+}>\mu_{-} \in \mathbb{R}$. If $w \in \mathbb{X}$ satisfies $\underline{L}_{\varphi} w=c(x) w$ in $\mathbb{R}^{n}$, with

$$
w(x) \geqslant 0 \text { in } \mathbb{R}^{n} \backslash U \text { and } c(x) \geqslant \kappa \text { if } x \in U,
$$

for some $\kappa>0$, then

$$
w(x) \geqslant 0 \quad \text { for any } x \in \mathbb{R}^{n} .
$$

H6. (Strong Maximum Principle for the difference equation): If $v \in \mathbb{X}$ satisfies $\underline{L}_{\varphi} v=f(\varphi+v)-f(\varphi)$ for some $\varphi \in \mathbb{X}$, and $v \geqslant 0$ in $\mathbb{R}^{n}$ with $v(0)=0$, then $v$ vanishes identically.

We remark that assumption (H1) is almost harmless (it boils down to the standard linearization procedure if the operator $L$ is differentiable). Similarly, (H2) is a very weak condition and it does not even require, in principle, a regularity theory for (1.1) (for instance one can suitably choose the space $\mathbb{X}$ in order to control enough derivatives of $u$ to obtain the required compactness).

Under the above assumptions, we may state our general result as follows:

Theorem 1.1. Let $u \in \mathbb{X}$ be a solution of (1.1), with $\|u\|_{C^{1, \beta}\left(\mathbb{R}^{n}\right)}$ finite, for some $\beta \in(0,1)$. Let L satisfy (H1)-(H6) and $f$ satisfy (1.4). Then $u$ possesses one-dimensional symmetry, that is, (1.5) holds.

Theorem 1.1 is motivated by a famous conjecture of Gibbons when $L$ is the Laplace operator (see $[14,48]$ ), which was motivated by the cosmological problem of detecting the shape of the interfaces which "separate" the different regions of the universe which possibly arose from the big bang. Such conjecture was proved independently and with different methods by $[4,8,36]$. See $[37,38]$ for the case of discontinuous nonlinearities. In [45] it is also shown that the uniform control of only one limit in (1.1) is enough to obtain that $u$ is one-dimensional under the additional assumption that $u$ is a minimal solution.

In this sense, Theorem 1.1 may be seen as a generalization of the results of $[4,8,36]$ to a more general class of operators. Such generalization is performed in order to apply Theorem 1.1 to concrete cases of interest. As an application, we consider the case in which $L$ is a fractional power of the Laplacian:

Theorem 1.2. Let $L=-(-\Delta)^{s}$, with $s \in(0,1)$. Let $f$ satisfy (1.4). If $u \in W^{3, \infty}\left(\mathbb{R}^{n}\right)$ is a solution of (1.1), then $u$ possesses one-dimensional symmetry.

We refer to $[54,63,64]$ for the basics of fractional Laplacian theory. We would like to recall that the fractional Laplacian is a very important operator, since it naturally surfaces in many different areas, such as: the thin obstacle problem [13], optimization [33], finance [27], phase transitions [1, 2, 24, 67], stratified materials [66], anomalous diffusion [55], crystal dislocation [57, 68], soft 
thin films [53], some models of semipermeable membranes and flame propagation [21], conservation laws [9], the ultrarelativistic limit of quantum mechanics [40], quasigeostrophic flows [20,56], multiple scattering [18, 31, 47], minimal surfaces $[22,28]$, materials science $[3]$, probability $[5,7,51,52,69]$, and water waves $[17,19,25,26,29,34,35,46,50,59,60,65,71,72]$.

When $s=\frac{1}{2}$, Theorem 1.2 was proven, by different methods, in [24], and an extension of that proof to any $s \in(0,1)$ is given in [23]. Also, we recall that in dimension $n=2$ the uniform limit assumption may be dropped in (1.1) and Theorem 1.2 still holds true for monotone solutions, as proved in $[23,67]$. The case $n=3$ has also been recently treated for some values of $s$, see [16], but many fundamental questions are still open.

Now, as another consequence of Theorem 1.1, we give a very general result on (possibly nonlinear) elliptic operators. For this, we denote by Sym ${ }^{n}$ the space of $(n \times n)$-symmetric matrices.

Theorem 1.3. Let $F=F(M, p) \in C^{1}\left(\operatorname{Sym}^{n} \times \mathbb{R}^{n}\right)$. Assume that there exists $\lambda \in C\left(\operatorname{Sym}^{n} \times \mathbb{R}^{n},(0,+\infty)\right)$ such that

$$
F(M+N, p)-F(M, p) \geqslant \lambda(M, p)\|N\|
$$

for any nonnegative definite $(n \times n)$-symmetric matrix $N$. Let $L u=F\left(D^{2} u, \nabla u\right)$. Let $f$ satisfy $(1.4)$ and $\beta \in(0,1)$. If $u \in C^{3, \beta}\left(\mathbb{R}^{n}\right)$ is a solution of $(1.1)$, then $u$ possesses one-dimensional symmetry.

We remark that condition (1.6) is an ellipticity assumption: compare, for instance, with Definition 2.1 on page 12 of [15] — we remark, in fact, that, by (1.6), $F$ is uniformly elliptic when restricted to any compact subset of $\operatorname{Sym}^{n} \times \mathbb{R}^{n}$ and, since $u$ is assumed to be smooth, then $F$ is evaluated in a bounded set of parameters where it is uniformly elliptic.

The application of Theorem 1.3 is very wide, since it comprises, for instance:

- The Laplace operator, with the choice

$$
F(M, p)=\operatorname{Tr} M,
$$

- Elliptic operators with constant coefficients, take

$$
F(M, p)=a_{i j} M_{i j}+b \cdot p,
$$

- Quasilinear operators, such as

$$
\begin{aligned}
& F(M, p)=\left(a+|p|^{2}\right)^{(m-2) / 2} \operatorname{Tr} M+(m-2)\left(a+|p|^{2}\right)^{(m-4) / 2} M_{i j} p_{i} p_{j}, \\
& \text { with } a>0 \text { and } m>1, \\
& \text { - The mean curvature operator }
\end{aligned}
$$

$$
F(M, p)=\left(1+|p|^{2}\right)^{-1 / 2} \operatorname{Tr} M-\left(1+|p|^{2}\right)^{-3 / 2} M_{i j} p_{i} p_{j},
$$


- The elliptic fully nonlinear operators.

We point out that the assumption that $u$ is smooth in Theorem 1.3 is not very restrictive, since it may be obtained in many cases via elliptic regularity theory once $u \in C^{1, \beta}\left(\mathbb{R}^{n}\right)$, see $[15]$ and references therein (in fact, a stronger regularity theory holds if $n=2$, see [58]).

We recall that a result similar to Theorem 1.3 for the uniformly elliptic fully nonlinear operators of the form $F(M, p)=F(M)$ has also been obtained in [62] by using the theory of viscosity solutions under the additional assumption on the existence of a suitable one-dimensional profile ${ }^{1}$. Thus, the viscosity setting of [62] and the classical one that we deal with here are related but different in spirit (though, under additional assumptions on the operator, viscosity solutions do become classical, see Chapters 8 and 9 in [15]).

It would be interesting to treat also the case of assumptions even more general than (H1)-(H6). For instance, it would be interesting to deal with operators in which elliptic singularities and degeneracies occur (see e.g. [45] and also $[32,41,70]$ for some results in this direction and [42] for related problems and further references). Other cases of interest that one would like to deal with are the subelliptic operators and the operators arising in hyperbolic geometry (see, e.g., $[6,10-12,43,44]$ for results in these frameworks, and also Section 2.8 of [42] for further details). Moreover, after this work was completed, we have received the interesting paper [30] in which related symmetry results have been obtained for viscosity solutions of some fully nonlinear PDEs of a special form.

The proof of Theorem 1.1 that we give makes use of the technique of $[36,39]$, suitably modified in order to comprise our general case. For this, in Section 2, we give an intermediate result based on monotonicity cones. In Section 3, we complete the proof of Theorem 1.1, while Theorems 1.2 and 1.3 are proven in Sections 4 and 5 respectively, by showing that the operators under consideration fulfill assumptions (H1)-(H6).

\section{A First Symmetry Result Via Monotonicity Cones}

The proof of Theorem 1.1 makes use of a first provisional statement, which goes as follows:

Lemma 2.1. Let $u \in \mathbb{X}$ be a bounded and uniformly Lipschitz solution of (1.1), with $L$ satisfying $(\mathrm{H} 1)-(\mathrm{H} 4)$ and $f$ satisfying (1.4). Assume also that there exists $a \in(0,1)$ such that

$$
\partial_{\nu} u(x)>0 \text { for any } x \in \mathbb{R}^{n} \text { and any } v=\left(v_{1}, \ldots, v_{n}\right) \in \mathrm{S}^{n-1} \text { with } v_{n} \geqslant a \text {. }
$$

\section{Then $u$ possesses one-dimensional symmetry.}

\footnotetext{
${ }^{1}$ It may be worth to remark that we do not need to assume that any one-dimensional profile exists. In fact, if no one-dimensional profile exists, our result may be seen as a non-existence result: namely, if we prove that the solution must be one-dimensional and no one-dimensional solution exists, then we have that there are no solutions at all.
} 
Of course, Lemma 2.1 is just Theorem 1.1 with the additional hypothesis on the monotonicity cone in (2.1): in Section 3 we will show that such additional assumption is, in fact, not needed and so we will be able to derive Theorem 1.1 from Lemma 2.1.

Proof. In order to prove Lemma 2.1, we show that

$$
\partial_{\nu} u(x)>0 \text { for any } x \in \mathbb{R}^{n} \text { and any } v=\left(v_{1}, \ldots, v_{n}\right) \in \mathrm{S}^{n-1} \text { with } v_{n}>0 \text {. }
$$

To prove (2.2), we take

$$
\underline{a}:=\inf \{a>0 \text { for which (2.1) holds }\} .
$$

If $\underline{a}=0$, then (2.2) is proved, so we assume, by contradiction, that

$$
\underline{a}>0 \text {. }
$$

Given $S>0$, we define

By construction,

$$
i_{S}:=\inf _{\substack{x^{\prime} \in \mathbb{R}^{n-1},\left|x_{n}\right| \leqslant S \\ v_{n} \geqslant \underline{a}}} \partial_{\nu} u\left(x^{\prime}, x_{n}\right) .
$$

$$
i_{S} \geqslant 0
$$

we claim that, in fact

$$
i_{S}>0 \text {. }
$$

To prove (2.6), we argue by contradiction and we suppose that there exist a sequence of $\nu^{(k)} \in S^{n-1}$ and $x^{(k)} \in \mathbb{R}^{n}$ with

$$
\left|x_{n}^{(k)}\right| \leqslant S,
$$

$v^{(k)} \geqslant \underline{a}$, and

$$
\lim _{k \rightarrow+\infty} \partial_{v^{(k)}} u\left(x^{(k)}\right)=0
$$

From (2.5),

$$
\partial_{\nu^{(k)}} u(x) \geqslant 0 \quad \text { for any } x \in \mathbb{R}^{n} \text {. }
$$

We define

$$
u^{(k)}(x):=u\left(x+x^{(k)}\right) .
$$


Then, (2.8) becomes

$$
\lim _{k \rightarrow+\infty} \nabla u^{(k)}(0) \cdot v^{(k)}=0 .
$$

Analogously, (2.9) writes

$$
\nabla u^{(k)}(x) \cdot v^{(k)} \geqslant 0 \quad \text { for any } x \in \mathbb{R}^{n} .
$$

Notice also that

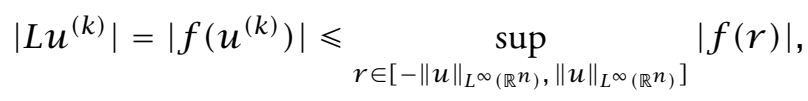

where (1.2) has been used. Thus, from (2.11), (2.12) and (H2), there exist $u^{(\infty)} \in$ $\mathbb{X}$ and $\nu^{\infty} \in S^{n-1}$, with

$$
v^{(\infty)} \geqslant \underline{a},
$$

such that

$$
\lim _{k \rightarrow+\infty} u^{(k)}(x)=u^{(\infty)}(x) \quad \text { for any } x \in \mathbb{R}^{n},
$$

and

$$
L u^{(\infty)}=f\left(u^{(\infty)}\right), \nabla u^{(\infty)} \cdot v^{(\infty)} \geqslant 0 \text { in } \mathbb{R}^{n}, \quad \text { with } \nabla u^{(\infty)}(0) \cdot v^{(\infty)}=0 .
$$

Therefore, by (H1), the function $v:=\partial_{v^{(\infty)}} u^{(\infty)} \in \tilde{\mathbb{X}}$ satisfies

$$
\tilde{L} v=f^{\prime}(u) v, v(x) \geqslant 0=v(0) \quad \text { for any } x \in \mathbb{R}^{n} .
$$

As a consequence, from (H4), $v$ vanishes identically. Accordingly,

$$
u^{(\infty)}\left(v^{(\infty)} t\right)-u^{(\infty)}\left(-v^{(\infty)} t\right)=\int_{-t}^{t} v\left(v^{(\infty)} s\right) \mathrm{d} s=0 \quad \text { for any } t \geqslant 0
$$

Recalling the uniform limit assumption in (1.1), we now take $M>0$ in such a way that

$$
u(x) \geqslant \frac{1}{2} \quad \text { if } x_{n} \geqslant M, \quad u(x) \leqslant-\frac{1}{2} \quad \text { if } x_{n} \leqslant-M .
$$

Then, recalling (2.7) and (2.10),

$$
u^{(k)}(x) \geqslant \frac{1}{2} \quad \text { if } x_{n} \geqslant M+S, \quad u^{(k)}(x) \leqslant-\frac{1}{2} \quad \text { if } x_{n} \leqslant-M-S .
$$


Hence, from (2.14),

$$
u^{(\infty)}(x) \geqslant \frac{1}{2} \quad \text { if } x_{n} \geqslant M+S, \quad u^{(\infty)}(x) \leqslant-\frac{1}{2} \quad \text { if } x_{n} \leqslant-M-S .
$$

We recall that, from (2.4) and (2.13), $v_{n}^{(\infty)} \geqslant \underline{a}>0$, so (2.16) implies that

$$
u^{(\infty)}\left(v^{(\infty)} t\right) \geqslant \frac{1}{2} \text { and } u^{(\infty)}\left(-v^{(\infty)} t\right) \leqslant-\frac{1}{2} \quad \text { if } t \geqslant \frac{M+S}{\underline{a}} .
$$

This and (2.15) give that

$$
0=u^{(\infty)}\left(v^{(\infty)} t\right)-u^{(\infty)}\left(-v^{(\infty)} t\right) \geqslant 1 .
$$

This contradiction proves (2.6).

Now, we use (1.4), to see that $f^{\prime}(r) \geqslant \kappa$, for a suitable $\kappa>0$, if $|r-1| \leqslant \eta^{\star}$, for a suitable $\eta^{\star} \in\left(0, \frac{1}{4}\right)$. Also, the uniform limit assumption in (1.1) enables us to take $M_{\star}>0$ such that $u(x) \geqslant 1-\eta^{\star}$ if $x_{n} \geqslant M_{\star}$ and $u(x) \leqslant-1+\eta^{\star}$ if $x_{n} \leqslant-M_{\star}$. We also define $c(x):=f^{\prime}(u(x))$. Hence, $c(x) \geqslant \kappa$ when $\left|x_{N}\right| \geqslant M_{\star}$. Let also

$$
\varepsilon:=\frac{i_{M_{\star}}}{2\left(1+\|\nabla u\|_{L^{\infty}\left(\mathbb{R}^{n}\right)}\right)} .
$$

Notice that $\varepsilon>0$, thanks to (2.6).

If $\left|x_{n}\right| \leqslant M_{\star}$ and $v \in \mathrm{S}^{n-1}$ with $v_{n} \in[\underline{a}-\varepsilon, \underline{a}]$, then

$$
\begin{aligned}
\partial_{\nu} u(x) & =\nabla u(x) \cdot v \geqslant \nabla u(x) \cdot\left(v^{\prime}, \underline{a}\right)-\left|\nabla u(x) \cdot\left(0, \underline{a}-v_{n}\right)\right| \\
& \geqslant i_{M_{\star}}-\|\nabla u\|_{L^{\infty}\left(\mathbb{R}^{n}\right)} \varepsilon \geqslant \frac{i_{M_{\star}}}{2}>0 .
\end{aligned}
$$

Therefore, by (H3), $\partial_{v} u(x) \geqslant 0$ for any $x \in \mathbb{R}^{n}$ and any $v \in \mathrm{S}^{n-1}$ with $v_{n} \in$ $[\underline{a}-\varepsilon, \underline{a}]$. In fact, by (H4), we see that $\partial_{\nu} u(x)>0$ for any $x \in \mathbb{R}^{n}$ and any $v \in \mathrm{S}^{n-1}$ with $v_{n} \in[\underline{a}-\varepsilon, \underline{a}]$. This is in contradiction with (2.3), and so it proves (2.2).

Then, from (2.2), by taking $\mu=-v$, we obtain that

$$
\partial_{\mu} u(x)<0 \quad \forall x \in \mathbb{R}^{n} \text { and } \forall \mu=\left(\mu_{1}, \ldots, \mu_{n}\right) \in \mathrm{S}^{n-1} \text { with } \mu_{n}<0 .
$$

By taking limits of $\nu_{n}$ and $\mu_{n}$ to 0 in (2.2) and (2.17), we deduce that

$$
\partial_{\omega} u(x)=0 \quad \forall x \in \mathbb{R}^{n} \text { and } \forall \omega=\left(\omega_{1}, \ldots, \omega_{n}\right) \in \mathrm{S}^{n-1} \text { with } \omega_{n}=0 .
$$

Hence, $\partial_{x_{1}} u(x)=\cdots=\partial_{x_{n-1}} u(x)=0$ for any $x \in \mathbb{R}^{n}$, which ends the proof of Lemma 2.1. 


\section{Proof of Theorem 1.1}

Some of the arguments needed to proof Theorem 1.1 will be appropriate modifications of the ones used in the proof of Lemma 2.1, by taking into account the difference operator $\underline{L}_{u}$ instead of the linearized operator $\tilde{L}$. In order to prove Theorem 1.1, first of all, we show that

$$
\partial_{n} u(x)>0 \text { for any } x \in \mathbb{R}^{n} .
$$

To prove (3.1), we take $h \geqslant 0$, we let

$$
\mathcal{T}_{h} u(x):=u\left(x+h e_{n}\right)-u(x)
$$

and we observe that

$$
f\left(u\left(x+h e_{n}\right)\right)-f(u(x))=c_{h}(x) \mathcal{T}_{h} u(x),
$$

where

$$
c_{h}(x):=\int_{0}^{1} f^{\prime}\left(t u(x)+(1-t) u\left(x+h e_{n}\right)\right) \mathrm{d} t .
$$

Now, recalling (1.4), we take $\delta \in\left(0, \frac{1}{2}\right)$ such that

$$
f^{\prime} \geqslant \kappa \quad \text { in }(-\infty,-1+\delta] \cup[1-\delta,+\infty) \text {, for some } \kappa>0 .
$$

Then, by the uniform limit assumption in (1.1), we take $M>0$ such that

$$
u(x) \geqslant 1-\delta \quad \text { if } x_{n} \geqslant M, \quad u(x) \leqslant-1+\delta \quad \text { if } x_{n} \leqslant-M .
$$

Now, we observe the following useful property:

$$
\text { if } x \in\left\{\mathcal{T}_{h} u<0\right\} \cap\left\{\left|x_{n}\right| \geqslant M\right\}, \quad \text { then } c_{h}(x) \geqslant \kappa \text {. }
$$

Indeed, on the one hand, if $x \in\left\{\mathcal{T}_{h} u<0\right\} \cap\left\{x_{n} \leqslant-M\right\}$,

$$
u\left(x+h e_{n}\right)<u(x) \leqslant-1+\delta
$$

and therefore

$$
t u(x)+(1-t) u\left(x+h e_{n}\right) \leqslant-1+\delta
$$

for any $x \in\left\{\mathcal{T}_{h} u<0\right\} \cap\left\{x_{n} \leqslant-M\right\}$ and $t \in[0,1]$. On the other hand, if $x \in\left\{\mathcal{T}_{h} u<0\right\} \cap\left\{x_{n} \geqslant M\right\}$, then $u(x)>u\left(x+h e_{n}\right) \geqslant 1-\delta$, and therefore

$$
t u(x)+(1-t) u\left(x+h e_{n}\right) \geqslant 1-\delta
$$


for any $x \in\left\{\mathcal{T}_{h} u<0\right\} \cap\left\{x_{n} \geqslant M\right\}$ and $t \in[0,1]$. From (3.3), (3.4), (3.7), and (3.8), we obtain that (3.6) holds true.

We claim that

$$
\text { if } h \geqslant 2 M, \quad \text { then } \mathcal{T}_{h} u(x)>0 \text { for any } x \in \mathbb{R}^{n} .
$$

To prove (3.9), fix $h \geqslant 2 M$ and let $U:=\left\{\mathcal{T}_{h} u<0\right\}$. Then,

$$
\text { if } x_{n}=-M, \mathcal{T}_{h} u(x) \geqslant \inf _{x_{n} \geqslant M} u(x)-\sup _{x_{n} \leqslant-M} u(x) \geqslant(1-\delta)-(-1+\delta)>0,
$$

and so

$$
U=U_{+} \cup U_{-},
$$

where $U_{+}$(respectively $U_{-}$) is an open set contained in the half-space $\left\{x_{n} \geqslant-M\right\}$ (respectively $\left\{x_{n} \leqslant-M\right\}$ ). Then, (3.2), (3.10) and (3.6), together with (H5), imply that if $h \geqslant 2 M$, then $\mathcal{T}_{h} u(x) \geqslant 0$ for any $x \in \mathbb{R}^{n}$. Hence, (3.9) follows from (H6).

Now, we define

$$
h_{0}:=\inf \left\{h>0 \text { such that } \mathcal{T}_{h} u(x)>0 \text { for any } x \in \mathbb{R}^{n} \text { with }\left|x_{n}\right| \leqslant M\right\} .
$$

Note that this definition is well-posed, due to (3.9).

We prove that

$$
h_{0}=0 .
$$

The proof of (3.11) is by contradiction. Suppose $h_{0}>0$. We have that

$$
u\left(x+\left(h_{0}+\varepsilon\right) e_{n}\right)-u(x) \geqslant 0 \text { for any } x \in\left\{\left|x_{n}\right| \leqslant M\right\} \text { and any } \varepsilon>0
$$

and

$$
u\left(x^{(k)}+\left(h_{0}-\varepsilon^{(k)}\right) e_{n}\right)-u\left(x^{(k)}\right) \leqslant 0 \quad \text { for some } x^{(k)} \in\left\{\left|x_{n}\right| \leqslant M\right\},
$$

where $\varepsilon^{(k)} \geqslant 0$ is an infinitesimal sequence. As a consequence,

$$
\mathcal{T}_{h_{0}} u(x)=\lim _{\varepsilon \rightarrow 0^{+}} u\left(x+\left(h_{0}+\varepsilon\right) e_{n}\right)-u(x) \geqslant 0 \quad \text { for any } x \in\left\{\left|x_{n}\right| \leqslant M\right\} .
$$

Therefore, recalling (3.6) and (H5),

$$
\mathcal{T}_{h_{0}} u(x) \geqslant 0 \quad \text { for any } x \in \mathbb{R}^{n} .
$$

Now we define $u^{(k)}(x):=u\left(x+x^{(k)}\right)$, and we deduce from (H2) that, up to a subsequence, $u^{(k)}$ approaches some $u^{(\infty)}$, with

$$
\underline{L}_{u}\left(\mathcal{T}_{h_{0}} u^{(\infty)}\right)=f\left(\mathcal{T}_{h_{0}} u^{(\infty)}+u\right)-f(u) .
$$


By (3.12), we see that $\mathcal{T}_{h_{0}} u^{(\infty)}(x) \geqslant 0$ for any $x \in \mathbb{R}^{n}$. Also,

$$
\begin{aligned}
\mathcal{T}_{h_{0}} u^{(\infty)}(0) & =\lim _{k \rightarrow+\infty} u\left(x^{(k)}+h_{0} e_{n}\right)-u\left(x^{(k)}\right) \\
& \leqslant \lim _{k \rightarrow+\infty} u\left(x^{(k)}+\left(h_{0}-\varepsilon^{(k)}\right) e_{n}\right)-u\left(x^{(k)}\right)+\varepsilon^{(k)}\|u\|_{C^{1, \beta}\left(\mathbb{R}^{n}\right)} \leqslant 0,
\end{aligned}
$$

hence $\mathcal{T}_{h_{0}} u^{(\infty)}(0)=0$. Consequently, by (H6), we get that $\mathcal{T}_{h_{0}} u^{(\infty)}$ vanishes identically. Therefore, $u^{(\infty)}\left(x+h_{0} e_{n}\right)=u^{(\infty)}(x)$ for any $x \in \mathbb{R}^{n}$ and so, by iterating,

$$
u^{(\infty)}\left(x+j h_{0} e_{n}\right)=u^{(\infty)}(x) \quad \text { for any } x \in \mathbb{R}^{n} \text { and any } j \in \mathbb{Z} .
$$

Now, if $j \in \mathbb{N} \cap\left[2 M / h_{0},+\infty\right)$, we have that $j h_{0}+x_{n}^{(k)} \geqslant M$ and $-j h_{0}+x_{n}^{(k)} \leqslant$ $-M$, so $u\left(j h_{0} e_{n}+x^{(k)}\right) \geqslant 1-\delta$ and $u\left(-j h_{0} e_{n}+x^{(k)}\right) \leqslant-1+\delta$. Then, for such a $j$,

$$
\begin{aligned}
2(1-\delta) & \geqslant \lim _{k \rightarrow+\infty} u\left(j h_{0} e_{n}+x^{(k)}\right)-u\left(-j h_{0} e_{n}+x^{(k)}\right) \\
& =u^{(\infty)}\left(j h_{0} e_{n}\right)-u^{(\infty)}\left(-j h_{0} e_{n}\right) .
\end{aligned}
$$

Since this contradicts (3.13), we have proved (3.11). That is, $\mathcal{T}_{h} u(x) \geqslant 0$ for any $x$ with $\left\{\left|x_{n}\right| \leqslant M\right\}$ and any $h \geqslant 0$. Consequently, by (3.6) and (H5), we deduce that $\mathcal{T}_{h} u(x) \geqslant 0$ for any $x \in \mathbb{R}^{n}$. Accordingly, $\partial_{n} u(x) \geqslant 0$ for any $x \in \mathbb{R}^{n}$, and then (3.1) follows from (H4).

Now we show that for any $S>0$ there exists $a(S) \in(0,1)$ such that

$$
\begin{array}{r}
\partial_{\nu} u(x)>0 \quad \text { for any } x \in \mathbb{R}^{n} \cap\left\{\left|x_{n}\right| \leqslant S\right\} \\
\text { and any } v=\left(v_{1}, \ldots, v_{n}\right) \in \mathrm{S}^{n-1} \text { with } v_{n} \geqslant a(S) .
\end{array}
$$

The proof of (3.14) is by contradiction. Suppose that, for a fixed $S$, there exist sequences $x^{(k)} \in\left\{\left|x_{n}\right| \leqslant S\right\}$ and $v^{(k)} \in \mathrm{S}^{n-1}$ such that $v_{n}^{(k)} \geqslant 1-(1 / k)$ and

$$
\partial_{v^{(k)}} u\left(x^{(k)}\right) \leqslant 0 .
$$

Let $u^{(k)}(x):=u\left(x+x^{(k)}\right)$. Notice that $v^{(k)}$ approaches $e_{n}$ for large $k$, therefore, by (H2), we obtain that, up to a subsequence, $u^{(k)}$ approaches some $u^{(\infty)}$ together with its derivative, with $L u^{(\infty)}=f\left(u^{(\infty)}\right)$ and $\tilde{L}\left(\partial_{n} u^{(\infty)}\right)=f^{\prime}\left(u^{(\infty)}\right) \partial_{n} u^{(\infty)}$. We remark that, by (3.1),

$$
\partial_{n} u^{(\infty)} \geqslant 0
$$

while, by (3.15),

$$
\partial_{n} u^{(\infty)}(0) \leqslant 0 .
$$


Accordingly, (H4) says that $\partial_{n} u^{(\infty)}$ vanishes identically. Thus, if $t-S$ is large enough (hence $t e_{n}-\left|x_{n}^{(k)}\right|$ is large enough), the uniform limit in (1.1) gives that

$$
\begin{aligned}
\frac{9}{10} & \leqslant \lim _{k \rightarrow+\infty} u\left(t e_{n}+x^{(k)}\right)=u^{(\infty)}\left(t e_{n}\right) \\
& =u^{(\infty)}\left(-t e_{n}\right)=\lim _{k \rightarrow+\infty} u\left(-t e_{n}+x^{(k)}\right) \\
& \leqslant-\frac{9}{10}
\end{aligned}
$$

This contradiction proves (3.14).

Now, recalling the definition of $M$ given in (3.4) and (3.5), in the notation of (3.14), we define

$$
a:=a(M)
$$

Then, as a consequence of (3.14), (H3), and (3.4), we have that $\partial_{\nu} u(x) \geqslant 0$ for any $x \in \mathbb{R}^{n}$, if $v_{n} \geqslant a$. Then, by (3.14) and (H4), we conclude that $\partial_{\nu} u(x)>0$ for any $x \in \mathbb{R}^{n}$, if $v_{n} \geqslant a$. That is, condition (2.1) holds true. Therefore, the proof of Theorem 1.1 is completed thanks to Lemma 2.1.

\section{Proof of Theorem 1.2}

We will deduce Theorem 1.2 from Theorem 1.1, in which $L=\tilde{L}=\underline{L}_{\varphi}:=$ $-(-\Delta)^{s}, \mathbb{X}:=W^{3, \infty}\left(\mathbb{R}^{n}\right)$, and $\tilde{\mathbb{X}}:=W^{2, \infty}\left(\mathbb{R}^{n}\right)$. For this, we need to check hypotheses (H1)-(H6). First, we claim that, if $u \in W^{3, \infty}\left(\mathbb{R}^{n}\right)$, then

$$
\partial_{\omega}\left(-(-\Delta)^{s} u\right)=-(-\Delta)^{s}\left(\partial_{\omega} u\right) .
$$

Indeed, (4.1) is obvious if $u$ belongs to the Schwartz class of rapidly decreasing functions, since, in this case, one can represent $(-\Delta)^{s}$ via a Fourier transform (see, for instance, $[54,63,64,69])$ and check $(4.1)$.

If, on the other hand, $u \in W^{3, \infty}\left(\mathbb{R}^{n}\right)$, we have that for any $h>0$,

$$
\begin{aligned}
& \frac{u(h \omega+y)+u(h \omega-y)-2 u(h \omega)}{|y|^{n+2 s}}-\frac{u(y)+u(-y)-2 u(0)}{|y|^{n+2 s}} \\
& \quad=\frac{u(h \omega+y)-u(y)+u(h \omega-y)-u(-y)-2 u(h \omega)+2 u(0)}{|y|^{n+2 s}} \\
& \quad \leqslant 5\|u\|_{W^{3, \infty}\left(\mathbb{R}^{n}\right)} h\left[|y|^{-(n+2 s)} \chi_{\mathbb{R}^{n} \backslash B_{1}}(y)+|y|^{2-(n+2 s)} \chi_{B_{1}}(y)\right] \in L^{1}\left(\mathbb{R}^{n}\right) .
\end{aligned}
$$


Thus, the Dominated Convergence Theorem gives that

$$
\begin{aligned}
& \partial_{\omega}\left(\int_{\mathbb{R}^{n}} \frac{u(x+y)+u(x-y)-2 u(x)}{|y|^{n+2 s}} \mathrm{~d} y\right)_{x=0} \\
& =\lim _{h \rightarrow 0^{+}} \int_{\mathbb{R}^{n}} \frac{u(h \omega+y)+u(h \omega-y)-2 u(h \omega)}{h|y|^{n+2 s}} \mathrm{~d} y \\
& \quad-\int_{\mathbb{R}^{n}} \frac{u(y)+u(-y)-2 u(0)}{h|y|^{n+2 s}} \mathrm{~d} y \\
& =\lim _{h \rightarrow 0^{+}} \int_{\mathbb{R}^{n}} \frac{u(h \omega+y)-u(y)+u(h \omega-y)-u(-y)-2 u(h \omega)+2 u(0)}{h|y|^{n+2 s}} \mathrm{~d} y \\
& =\int_{\mathbb{R}^{n}} \frac{\partial_{\omega} u(y)+\partial_{\omega} u(-y)-2 \partial_{\omega} u(0)}{|y|^{n+2 s}} \mathrm{~d} y .
\end{aligned}
$$

Thus, via the integral representation of the fractional Laplacian (see [54, 63, 64, 69]), the above identity reads

$$
\partial_{\omega}\left(-(-\Delta)^{s} u(x)\right)_{x=0}=-\left((-\Delta)^{s}\left(\partial_{\omega} u\right)\right)(0),
$$

which proves (4.1) at $x=0$ (and analogously at any point).

Then, hypothesis (H1) follows from (4.1). Hypothesis (H2) follows from the fact that $\mathbb{X}=W^{3, \infty}$, using the Theorem of Ascoli and the integral representation of the fractional Laplacian. We now prove (H3), by arguing by contradiction. We suppose that

$$
i:=\inf _{\mathbb{R}^{n}} w<0,
$$

and we take $x^{(k)} \in \mathbb{R}^{n}$ such that

$$
\lim _{k \rightarrow+\infty} w\left(x^{(k)}\right)=i<0 .
$$

In particular, we may suppose that $w\left(x^{(k)}\right) \leqslant i / 2<0$, and therefore $x^{(k)} \in$ $\left\{\left|x_{n}\right| \geqslant M\right\}$, so $c\left(x^{(k)}\right) \geqslant \kappa>0$. Thus, if we set $w^{(k)}(x):=w\left(x+x^{(k)}\right)$, we see that

$$
-(-\Delta)^{s} w^{(k)}(x)=-(-\Delta)^{s} w\left(x+x^{(k)}\right)=c\left(x+x^{(k)}\right) w\left(x+x^{(k)}\right) .
$$

In particular,

$$
\begin{aligned}
C(n, s) \int_{\mathbb{R}^{n}} \frac{w^{(k)}(y)-w^{(k)}(0)}{|y|^{n+2 s}} & =-(-\Delta)^{s} w^{(k)}(0) \\
& =c\left(x^{(k)}\right) w\left(x^{(k)}\right) \leqslant \frac{\kappa i}{2},
\end{aligned}
$$


for a suitable $C(n, s)>0$. In the first equality in (4.2), we have used one of the classical representations of the fractional Laplacian, see, e.g., [54, 63, 64, 69] for details.

Since $w \in \tilde{\mathbb{X}}:=W^{2, \infty}\left(\mathbb{R}^{n}\right)$, we have that $w^{(k)}$ converges locally uniformly to some $w^{(\infty)}$, up to a subsequence, due to Theorem of Ascoli, and so, by taking the limit in (4.2), we have

$$
C(n, s) \int_{\mathbb{R}^{n}} \frac{w^{(\infty)}(y)-w^{(\infty)}(0)}{|y|^{n+2 s}} \leqslant \frac{\kappa i}{2} .
$$

On the other hand,

$$
\begin{aligned}
w^{(\infty)}(0) & =\lim _{k \rightarrow+\infty} w^{(k)}(0)=\lim _{k \rightarrow+\infty} w\left(x^{(k)}\right) \\
& =\inf _{\mathbb{R}^{n}} w \leqslant w\left(y+x^{(k)}\right)=w^{(k)}(y)
\end{aligned}
$$

for any $y \in \mathbb{R}^{n}$, and so

$$
w^{(\infty)}(0) \leqslant w^{(\infty)}(y)
$$

for any $y \in \mathbb{R}^{n}$. As a consequence, (4.3) gives that

$$
0 \leqslant C(n, s) \int_{\mathbb{R}^{n}} \frac{w^{(\infty)}(y)-w^{(\infty)}(0)}{|y|^{n+2 s}} \leqslant \frac{\kappa i}{2}<0 .
$$

This contradiction proves $(\mathrm{H} 3)$.

Take now $v$ as requested in (H4): then, the integral representation of the fractional Laplacian gives that, for a suitable $C(n, s)>0$,

$$
\begin{aligned}
0 & =f^{\prime}(u(0)) v(0)=-(-\Delta)^{s} v(0) \\
& =C(n, s) \int_{\mathbb{R}^{n}} \frac{v(y)-v(0)}{|y|^{n+2 s}} \mathrm{~d} y=C(n, s) \int_{\mathbb{R}^{n}} \frac{v(y)}{|y|^{n+2 s}} \mathrm{~d} y,
\end{aligned}
$$

with the integral taken in the Cauchy principal value sense. Since $v \geqslant 0,(4.5)$ implies that $v$ is identically zero, thus checking $(\mathrm{H} 4)$.

The proof of (H5) (respectively (H6)) is analogous to the one of $(\mathrm{H} 3)$ (respectively (H4)): just take $U$ instead of $\left\{\left|x_{n}\right| \geqslant M\right\}$ (respectively $f(u+v)-f(u)$ instead of $\left.f^{\prime}(u) v\right)$. The proof of Theorem 1.2 is thus complete.

\section{Proof of Theorem 1.3}

We take $\mathbb{X}:=C^{3, \beta}\left(\mathbb{R}^{n}\right)$ and $\widetilde{\mathbb{X}}:=C^{2, \beta}\left(\mathbb{R}^{n}\right)$. Notice that, for any $v \in \mathbb{\mathbb { X }}$,

$$
f(u+v)-f(u)=\underline{c} v, \quad \text { with } \underline{c}(x):=\int_{0}^{1} f^{\prime}(u(x)+t v(x)) \mathrm{d} t .
$$


Also,

$$
\begin{aligned}
\tilde{L} v= & \sum_{i, j=1}^{n} \tilde{a}_{i j} \partial_{i j}^{2} v+\tilde{b} \cdot \nabla v, \\
\underline{L}_{u} v= & F\left(D^{2} u+D^{2} v, \nabla u+\nabla v\right)-F\left(D^{2} u, \nabla u+\nabla v\right) \\
& +F\left(D^{2} u, \nabla u+\nabla v\right)-F\left(D^{2} u, \nabla u\right) \\
= & \sum_{i, j=1}^{n} \underline{a}_{i j} \partial_{i j}^{2} v+\underline{b} \cdot \nabla v,
\end{aligned}
$$

with

$$
\begin{aligned}
\tilde{a}_{i j}(x) & :=\frac{\partial F}{\partial M_{i j}}\left(D^{2} u(x), \nabla u(x)\right), \\
\tilde{b}(x) & :=\frac{\partial F}{\partial p}\left(D^{2} u(x), \nabla u(x)\right), \\
\underline{a}_{i j}(x) & :=\int_{0}^{1} \frac{\partial F}{\partial M_{i j}}\left(D^{2} u(x)+t D^{2} v(x), \nabla u(x)\right) \mathrm{d} t,
\end{aligned}
$$

and

$$
\underline{b}(x):=\int_{0}^{1} \frac{\partial F}{\partial p}\left(D^{2} u(x), \nabla u(x)+t \nabla v(x)\right) \mathrm{d} t .
$$

In this way, (H1) is obviously satisfied and (H2) is a consequence of the Theorem of Ascoli. We observe that, by construction

$$
\tilde{a}_{i j}, \underline{a}_{i j}, \tilde{b}, \underline{b}, \underline{c} \in C^{0, \beta}\left(\mathbb{R}^{n}\right) \subset L_{\mathrm{loc}}^{\infty}\left(\mathbb{R}^{n}\right) .
$$

Moreover, from (1.6)

$$
\sum_{i, j=1}^{n} \frac{\partial F}{\partial M_{i j}}(M, p) N_{i j}=\lim _{s \rightarrow 0^{+}} \frac{F(M+s N, p)-F(M, p)}{s} \geqslant \lambda(M, p)\|N\|,
$$

for any nonnegative definite matrix $N$. In particular, given any $\xi \in \mathbb{R}^{n}$, taking $N_{i j}:=\xi_{i} \xi_{j}$,

$$
\begin{aligned}
\sum_{i, j=1}^{n} \frac{\partial F}{\partial M_{i j}}(M, p) \xi_{i} \xi_{j} & \geqslant \lambda(M, p) \sqrt{\sum_{i, j=1}^{n}\left(\xi_{i} \xi_{j}\right)^{2}} \geqslant \lambda(M, p) \sqrt{\sum_{i=1}^{n}\left(\xi_{i} \xi_{i}\right)^{2}} \\
& \geqslant \frac{\lambda(M, p)}{n^{2}}\|\xi\|^{2}
\end{aligned}
$$


Therefore, given any $R>0$, there exists $\lambda_{R, u, v}^{\star}>0$ such that $\inf _{\substack{x \in B_{R} \\ \tau, \sigma \in[0,1]}} \sum_{i, j} \frac{\partial F}{\partial M_{i j}}\left(D^{2} u(x)+\tau D^{2} v(x), \nabla u(x)+\sigma \nabla v(x)\right) \xi_{i} \xi_{j} \geqslant \lambda_{R, u, v}^{\star}\|\xi\|^{2}$.

As a consequence, for any $\xi \in \mathbb{R}^{n}$,

$$
\inf _{x \in B_{R}} \sum_{i, j=1}^{n} \tilde{a}_{i j} \xi_{i} \xi_{j} \geqslant \lambda_{R, u, v}^{\star}\|\xi\|^{2} \quad \text { and } \quad \inf _{x \in B_{R}} \sum_{i, j=1}^{n} \underline{a}_{i j} \xi_{i} \xi_{j} \geqslant \lambda_{R, u, v}^{\star}\|\xi\|^{2} .
$$

In particular,

$$
\sum_{i, j=1}^{n} \tilde{a}_{i j} \xi_{i} \xi_{j} \geqslant 0 \text { and } \sum_{i, j=1}^{n} \underline{a}_{i j} \xi_{i} \xi_{j} \geqslant 0 .
$$

Then, (H4) and (H6) are a consequence of (5.1), (5.2), (5.3), (5.4), and Hopf Strong Maximum Principle (see, for instance, [49] or Theorem 2.1.2 of [61]). Therefore, in order to complete the proof of Theorem 1.3, it remains to prove (H3) and (H5) (and then invoke Theorem 1.1).

We prove (H5) (the proof of (H3) is completely analogous). Suppose, by contradiction, that the conditions on $w$ in (H5) hold, but

$$
i:=\inf _{\mathbb{R}^{n}} w<0 .
$$

We take $x^{(k)} \in \mathbb{R}^{n}$ such that

$$
\lim _{k \rightarrow+\infty} w\left(x^{(k)}\right)=i<0 .
$$

In particular, we may suppose that $w\left(x^{(k)}\right) \leqslant i / 2<0$, and therefore $x^{(k)} \in U$, and so $c\left(x^{(k)}\right) \geqslant \kappa>0$.

We set $w^{(k)}(x):=w\left(x+x^{(k)}\right)$ and we use the definition of $\tilde{X}$ and the Theorem of Ascoli to obtain, up to a subsequence, that $w^{(k)}$ approaches some $w^{(\infty)}$ locally uniformly together with two derivatives. This also gives the convergence of the coefficients $\underline{a}_{i j}=\underline{a}_{i j}^{(k)}$ and $\underline{b}=\underline{b}^{(k)}$ obtained for $w^{(k)}$ via (5.2) to suitable $\underline{a}_{i j}^{(\infty)}$ and $\underline{b}^{(\infty)}$. Notice that, from (5.5), we have

$$
\sum_{i, j=1}^{n} \underline{a}_{i j}^{(\infty)} \xi_{i} \xi_{j} \geqslant 0 \quad \text { for any } \xi \in \mathbb{R}^{n} .
$$

Also, 0 is a minimum for $w^{(\infty)}$ (see the computation in (4.4)), and so $\nabla w^{(\infty)}(0)=$ 0 and $D^{2} w^{(\infty)}(0)$ is nonnegative definite. 
As a consequence, recalling (5.6),

$$
\begin{aligned}
\frac{\kappa i}{2} & \geqslant \lim _{k \rightarrow+\infty} c\left(x^{(k)}\right) w\left(x^{(k)}\right)=\lim _{k \rightarrow+\infty} \underline{L}_{u} w\left(x^{(k)}\right) \\
& =\lim _{k \rightarrow+\infty} \sum_{i, j=1}^{n} \underline{a}_{i j}^{(k)} \partial_{i j}^{2} w\left(x^{(k)}\right)+\underline{b}^{(k)} \cdot \nabla w\left(x^{(k)}\right) \\
& =\lim _{k \rightarrow+\infty} \sum_{i, j=1}^{n} \underline{a}_{i j}^{(k)} \partial_{i j}^{2} w^{(k)}(0)+\underline{b}^{(k)} \cdot \nabla w^{(k)}(0) \\
& =\sum_{i, j=1}^{n} \underline{a}_{i j}^{(\infty)} \partial_{i j}^{2} w^{(\infty)}(0)+\underline{b}^{(\infty)} \cdot \nabla w^{(\infty)}(0) \\
& \geqslant 0 .
\end{aligned}
$$

Since $i<0$, this is a contradiction and it proves (H5). The proof of Theorem 1.3 is thus completed.

Acknowledgements. It is a pleasure to thank Isabeau Birindelli for very nice and instructive conversations. The work of EV has been supported by FIRB Project Analysis and Beyond and GNAMPA Project Equazioni nonlineari su varietà: proprietà qualitative e classificazione delle soluzioni.

\section{REFERENCES}

[1] G. Alberti and G. BelletTini, A nonlocal anisotropic model for phase transitions. I. The optimal profile problem, Math. Ann. 310 (1998), no. 3, 527-560. http://dx.doi.org/10.1007/s002080050159. MR1612250 (2000b:82013)

[2] G. Alberti, G. Bouchitté, and P. SeppeCher, Phase transition with the line-tension effect, Arch. Rational Mech. Anal. 144 (1998), no. 1, 1-46. MR1657316 (99j:76104)

[3] P.W. BATES, On some nonlocal evolution equations arising in materials science, Nonlinear Dynamics and Evolution Equations, Fields Inst. Commun., vol. 48, Amer. Math. Soc., Providence, RI, 2006, pp. 13-52. MR2223347 (2007g:35097)

[4] M.T. BARLOW, R.F. BASS, and C. GUI, The Liouville property and a conjecture of De Giorgi, Comm. Pure Appl. Math. 53 (2000), no. 8, 1007-1038.

http://dx.doi.org/1 0.1002/1097-0312(200008)53:8\%3C1007::AID-CPA3\%3E3.3.CO;2-L. MR1755949 (2001m:35095)

[5] J. Bertoin, Lévy Processes, Cambridge Tracts in Mathematics, vol. 121, Cambridge University Press, Cambridge, 1996. MR1406564 (98e:60117)

[6] I. Birindelli, F. Ferrari, and E. ValdinOCI, Semilinear PDEs in the Heisenberg group: the role of the right invariant vector fields, Nonlinear Anal. 72 (2010), no. 2, 987-997. http://dx.doi.org/10.1016/j.na.2009.07.039. MR2579363 (2010k:35506)

[7] T. BOJDECKI and L.G. GOROSTIZA, Fractional Brownian motion via fractional Laplacian, Statist. Probab. Lett. 44 (1999), no. 1, 107-108. http://dx.doi.org/10.1016/S0167-7152(99)00014-0. MR1706362 (2000f:43005)

[8] H. Berestycki, F. Hamel, and R. Monneau, One-dimensional symmetry of bounded entire solutions of some elliptic equations, Duke Math. J. 103 (2000), no. 3, 375-396. http://dx.doi.org/10.1215/S0012-7094-00-10331-6. MR1763653 (2001j:35069) 
[9] P. BILER, G. KARCH, and W.A. WOYCZYŃSKI, Critical nonlinearity exponent and self-similar asymptotics for Lévy conservation laws, Ann. Inst. H. Poincaré Anal. Non Linéaire 18 (2001), no. 5, 613-637. http://dx.doi.org/10.1016/S0294-1449(01)00080-4. MR1849690 (2002f:35035)

[10] I. BIRINDELLI and E. LANCONELLI, A note on one dimensional symmetry in Carnot groups, Atti Accad. Naz. Lincei Cl. Sci. Fis. Mat. Natur. Rend. Lincei (9) Mat. Appl. 13 (2002), no. 1, 17-22 (English, with English and Italian summaries). MR1949145 (2003j:35008)

[11] _ A negative answer to a one-dimensional symmetry problem in the Heisenberg group, Calc. Var. Partial Differential Equations 18 (2003), no. 4, 357-372. http://dx.doi.org/10.1007/s00526-003-0194-0. MR2020366 (2004j:35059)

[12] I. BIRINDELli and R. MAZZEO, Symmetry for solutions of two-phase semilinear elliptic equations on hyperbolic space, Indiana Univ. Math. J. 58 (2009), no. 5, 2347-2368. http://dx.doi.org/10.1512/iumj.2009.58.3714. MR2583503

[13] L.A. CAFFARELLI, Further regularity for the Signorini problem, Comm. Partial Differential Equations 4 (1979), no. 9, 1067-1075. http://dx.doi.org/10.1080/03605307908820119. MR542512 (80i:35058)

[14] G. Carbou, Unicité et minimalité des solutions d'une équation de Ginzburg-Landau, Ann. Inst. H. Poincaré Anal. Non Linéaire 12 (1995), no. 3, 305-318 (French, with English and French summaries). MR1340266 (96m:35078)

[15] L.A. Caffarelli and X. Cabré, Fully Nonlinear Elliptic Equations, American Mathematical Society Colloquium Publications, vol. 43, American Mathematical Society, Providence, RI, 1995. MR1351007 (96h:35046)

[16] X. CABRÉ and E. CINTI, Energy estimates and 1-D symmetry for nonlinear equations involving the half-Laplacian, Discrete Contin. Dyn. Syst. 28 (2010), no. 3, 1179-1206. http://dx.doi.org/10.3934/dcds.2010.28.1179. MR2644786 (2011c:35139)

[17] W. CRAIG and M.D. GROVES, Hamiltonian long-wave approximations to the water-wave problem, Wave Motion 19 (1994), no. 4, 367-389. http://dx.doi.org/10.1016/0165-2125(94)90003-5. MR1285131 (95h:76008)

[18] D. COlTON and R. KRESS, Inverse Acoustic and Electromagnetic Scattering Theory, 2nd ed., Applied Mathematical Sciences, vol. 93, Springer-Verlag, Berlin, 1998. MR1635980 (99c:35181)

[19] W. CRAIG and D.P. NICHOLLS, Travelling two and three dimensional capillary gravity water waves, SIAM J. Math. Anal. 32 (2000), no. 2, 323-359.

http://dx.doi.org/10.1137/S0036141099354181. MR1781220 (2002c:76016)

[20] D. CordobA, Nonexistence of simple hyperbolic blow-up for the quasi-geostrophic equation, Ann. of Math. (2) 148 (1998), no. 3, 1135-1152. http://dx.doi.org/10.2307/121037. MR1670077 (2000j:76020)

[21] L.A. CafFarelli, J.-M. RoQUejOFFre, and Y. Sire, Free boundaries with fractional Laplacians (2009), preprint.

[22] L.A. Caffarelli, J.-M. Roquejoffre, and V.O. SaVin, Nonlocal minimal surfaces, Comm. Pure Appl. Math. 63 (2010), no. 9, 1111-1144. MR2675483

[23] X. CABRÉ and Y. SIRE, Semilinear equations with fractional Laplacians (2010), preprint.

[24] X. CABRÉ and J. SOLÀ-MORALES, Layer solutions in a half-space for boundary reactions, Comm. Pure Appl. Math. 58 (2005), no. 12, 1678-1732. http://dx.doi.org/10.1002/cpa.20093. MR2177165 (2006i:35116)

[25] W. CRAIG, C. SUlEM, and P.-L. SUlEM, Nonlinear modulation of gravity waves: a rigorous approach, Nonlinearity 5 (1992), no. 2, 497-522. http://dx.doi.org/10.1088/0951-7715/5/2/009. MR1158383 (93k:76012)

[26] W. CRAig, U. SCHANZ, and C. Sulem, The modulational regime of three-dimensional water waves and the Davey-Stewartson system, Ann. Inst. H. Poincaré Anal. Non Linéaire 14 (1997), no. 5, 615-667 (English, with English and French summaries).

http://dx.doi.org/10.1016/S0294-1449(97)80128-X. MR1470784 (99i:35144) 
[27] R. CONT and P. TAnKov, Financial Modelling with Jump Processes, Chapman \& Hall/CRC Financial Mathematics Series, Chapman \& Hall/CRC, Boca Raton, FL, 2004. MR2042661 (2004m:91004)

[28] L.A. CAFFARElli and E. VAldinOCI, Uniform estimates and limiting arguments for nonlocal minimal surfaces, Calc. Var. Partial Differential Equations 41 (2011), no. 1-2, 203-240. http://dx.doi.org/10.1007/s00526-010-0359-6. MR2782803

[29] W. CRAIG and P.A. WORFOLK, An integrable normal form for water waves in infinite depth, Phys. D 84 (1995), no. 3-4, 513-531. http://dx.doi.org/10.1016/0167-2789(95)00067-E. MR1336546 (96g:76007)

[30] F. Demengel and I. BiRindelli, One-dimensional symmetry for solutions of Allen Cahn fully nonlinear equations, Symmetry for Elliptic PDEs, Contemp. Math., vol. 528, Amer. Math. Soc., Providence, RI, 2010, pp. 1-15. MR2759031

[31] J.J. DUISTERMAAT and V.W. GUILLEMIN, The spectrum of positive elliptic operators and periodic bicharacteristics, Invent. Math. 29 (1975), no. 1, 39-79. http://dx.doi.org/10.1007/BF01405172. MR0405514 (53 \#9307)

[32] D. DANIELLI and N. GAROFALO, Properties of entire solutions of non-uniformly elliptic equations arising in geometry and in phase transitions, Calc. Var. Partial Differential Equations 15 (2002), no. 4, 451-491. http://dx.doi.org/10.1007/s005260100133. MR1942128 (2003j:35086)

[33] G. DuVAUT and J.-L. LIONS, Inequalities in Mechanics and Physics, Grundlehren der Mathematischen Wissenschaften, Band 219, Springer-Verlag, Berlin, 1976. Translated from the French by C.W. John. MR0521262 (58 \#25191)

[34] R. DE La Llave and P. PANAYOTAROS, Gravity waves on the surface of the sphere, J. Nonlinear Sci. 6 (1996), no. 2, 147-167. http://dx.doi.org/10.1007/s003329900007. MR1381401 (97f:76011)

[35] R. DE LA LLAVE and E. VALDINOCI, Symmetry for a Dirichlet-Neumann problem arising in water waves, Math. Res. Lett. 16 (2009), no. 5, 909-918. MR2576707 (2011b:35350)

[36] A. FARINA, Symmetry for solutions of semilinear elliptic equations in $\mathbb{R}^{N}$ and related conjectures, Ricerche Mat. 48 (1999), no. suppl., 129-154. Papers in memory of Ennio De Giorgi (Italian). MR1765681 (2001h:35056)

[37] _ Propriétés de monotonie et de symétrie unidimensionnelle pour les solutions de $\Delta u+$ $f(u)=0$ avec des fonctions $f$ éventuellement discontinues, C. R. Acad. Sci. Paris Sér. I Math. 330 (2000), no. 11, 973-978 (French, with English and French summaries). http://dx.doi.org/10.1016/S0764-4442(00)00305-0. MR1779689 (2001d:35053)

[38] _ Monotonicity and one-dimensional symmetry for the solutions of $\Delta u+f(u)=0$ in $\mathbb{R}^{N}$ with possibly discontinuous nonlinearity, Adv. Math. Sci. Appl. 11 (2001), no. 2, 811834. MR1907468 (2003e:35086)

[39] , Rigidity and one-dimensional symmetry for semilinear elliptic equations in the whole of $\mathbb{R}^{N}$ and in half spaces, Adv. Math. Sci. Appl. 13 (2003), no. 1, 65-82. MR2002396 (2004m:35078)

[40] C. FEFFERMAN and R. DE LA LLAVE, Relativistic stability of matter. I, Rev. Mat. Iberoamericana 2 (1986), no. 1-2, 119-213. MR864658 (88g:81155)

[41] A. Farina, B. SCIUnZI, and E. VAldinoCI, Bernstein and De Giorgi type problems: new results via a geometric approach, Ann. Sc. Norm. Super. Pisa Cl. Sci. (5) 7 (2008), no. 4, $741-$ 791. MR2483642 (2009j:58020)

[42] A. FARINA and E. VALDINOCI, The state of the art for a conjecture of De Giorgi and related problems, Recent Progress on Reaction-diffusion Systems and Viscosity Solutions, World Sci. Publ., Hackensack, NJ, 2009, pp. 74-96. http://dx.doi.org/10.1142/9789812834744_0004. MR2528756 (2011d:35156)

[43] F. Ferrari and E. VAldinOCI, A geometric inequality in the Heisenberg group and its applications to stable solutions of semilinear problems, Math. Ann. 343 (2009), no. 2, 351-370. http://dx.doi.org/10.1007/s00208-008-0274-8. MR2461257 (2009j:35044) 
[44] - Geometric PDEs in the Grushin plane: weighted inequalities and flatness of level sets, Int. Math. Res. Not. IMRN 22 (2009), 4232-4270. http://dx.doi.org/10.1093/imrn/rnp088. MR2552303 (2010j:35025)

[45] A. FARINA and E. VALDINOCI, 1D symmetry for solutions of semilinear and quasilinear elliptic equations, Trans. Amer. Math. Soc. 363 (2011), no. 2, 579-609. http://dx.doi.org/10.1090/S0002-9947-2010-05021-4. MR2728579 (2011j:35081)

[46] G.K. GÄCHTER and M.J. GROTE, Dirichlet-to-Neumann map for three-dimensional elastic waves, Wave Motion 37 (2003), no. 3, 293-311. http://dx.doi.org/10.1016/S0165-2125(02)00091-4. MR1957289 (2003m:74062)

[47] M.J. GROTE and C. KIRSCH, Dirichlet-to-Neumann boundary conditions for multiple scattering problems, J. Comput. Phys. 201 (2004), no. 2, 630-650. http://dx.doi.org/10.1016/j.jcp.2004.06.012. MR2100517 (2005h:65184)

[48] G.W. GIBBONS and P.K. TOWNSEND, Bogomol'nyi equation for intersecting domain walls, Phys. Rev. Lett. 83 (1999), no. 9, 1727-1730. http://dx.doi.org/10.1 103/PhysRevLett.83.1727.

[49] D. Gilbarg and N.S. Trudinger, Elliptic Partial Differential Equations of Second Order, Classics in Mathematics, Springer-Verlag, Berlin, 2001. Reprint of the 1998 edition. MR1814364 (2001k:35004)

[50] B. HU and D.P. NiCHOLLS, Analyticity of Dirichlet-Neumann operators on Hölder and Lipschitz domains, SIAM J. Math. Anal. 37 (2005), no. 1, 302-320 (electronic). http://dx.doi.org/10.1137/S0036141004444810. MR2176934 (2006f:35054)

[51] K. ITO, Lectures on Stochastic Processes, 2nd ed., Tata Institute of Fundamental Research Lectures on Mathematics and Physics, vol. 24, Distributed for the Tata Institute of Fundamental Research, Bombay, 1984. Notes by K. Muralidhara Rao. MR759892 (86f:60049)

[52] B. JOURDAIN, S. MÉLÉARD, and W.A. WOYCZYNSKI, A probabilistic approach for nonlinear equations involving the fractional Laplacian and a singular operator, Potential Anal. 23 (2005), no. 1, 55-81. http://dx.doi.org/10.1007/s111118-004-3264-9. MR2136209 (2006a:35327)

[53] M. KURZKE, A nonlocal singular perturbation problem with periodic well potential, ESAIM Control Optim. Calc. Var. 12 (2006), no. 1, 52-63 (electronic). http://dx.doi.org/10.1051/cocv:2005037. MR2192068 (2006j:49022)

[54] N.S. LANDKOF, Foundations of Modern Potential Theory, Die Grundlehren der mathematischen Wissenschaften, Band 180, Springer-Verlag, New York, 1972. Translated from the Russian by A.P. Doohovskoy. MR0350027 (50 \#2520)

[55] R. METZLER and J. KLAFTER, The random walk's guide to anomalous diffusion: a fractional dynamics approach, Phys. Rep. 339 (2000), no. 1, 77. http://dx.doi.org/10.1016/S0370-1573(00)00070-3. MR1809268 (2001k:82082)

[56] A.J. MAJDA and E.G. TABAK, A two-dimensional model for quasigeostrophic flow: comparison with the two-dimensional Euler flow, Phys. D 98 (1996), no. 2-4, 515-522, Nonlinear Phenomena in Ocean Dynamics (Los Alamos, NM, 1995). http://dx.doi.org/1 0.1016/0167-2789(96)001 14-5. MR1422288 (97g:86005)

[57] F.R.N. NABARRO, The mathematical theory of stationary dislocations, Advances in Physics 1 (1952), no. 3, 269-394 (1 plate). http://dx.doi.org/10.1080/00018735200101211. MR0068433 (16,883e)

[58] L. NirenberG, On nonlinear elliptic partial differential equations and Hölder continuity, Comm. Pure Appl. Math. 6 (1953), no. 1, 103-156; addendum, 395. http://dx.doi.org/10.1002/cpa.3160060105. MR0064986 (16,367c)

[59] P.I. NAUMKIN and I.A. SHISHMARËV, Nonlinear Nonlocal Equations in the Theory of Waves, Translations of Mathematical Monographs, vol. 133, American Mathematical Society, Providence, RI, 1994. Translated from the Russian manuscript by B. Gommerstadt. MR1261868 (94m:35230) 
[60] D.P. NICHOLLS and M. TABER, Joint analyticity and analytic continuation of Dirichlet-Neumann operators on doubly perturbed domains, J. Math. Fluid Mech. 10 (2008), no. 2, 238-271. http://dx.doi.org/10.1007/s00021-006-0231-9. MR2411413 (2010f:76011)

[61] P. PUCCI and J. SERrin, The Maximum Principle, Progress in Nonlinear Differential Equations and Their Applications, vol. 73, Birkhäuser Verlag, Basel, 2007. MR2356201 (2008m:35001)

[62] V.O. SAVIN, Entire solutions to a class of fully nonlinear elliptic equations, Ann. Sc. Norm. Super. Pisa Cl. Sci. (5) 7 (2008), no. 3, 369-405. MR2466434 (2010c:35052)

[63] L. SilVESTRE, Regularity of the obstacle problem for a fractional power of the Laplace operator, Ph.D. thesis, University of Texas at Austin, 2005.

[64] E.M. STEIn, Singular Integrals and Differentiability Properties of Functions, Princeton Mathematical Series, vol. 30, Princeton University Press, Princeton, N.J., 1970. MR0290095 (44 \#7280)

[65] J.J. STOKeR, Water Waves: The Mathematical Theory with Applications, Pure and Applied Mathematics, vol. IV, Interscience Publishers, Inc., New York, 1957. MR0103672 (21 \#2438)

[66] V.O. SAVIN and E. VALDINOCI, Elliptic PDEs with fibered nonlinearities, J. Geom. Anal. 19 (2009), no. 2, 420-432. http://dx.doi.org/10.1007/s12220-008-9064-5. MR2481968 (2009m:35165)

[67] Y. SIRE and E. VALDINOCI, Fractional Laplacian phase transitions and boundary reactions: a geometric inequality and a symmetry result, J. Funct. Anal. 256 (2009), no. 6, 1842-1864. http://dx.doi.org/10.1016/j.jfa.2009.01.020. MR2498561 (2010c:35201)

[68] J.F. Toland, The Peierls-Nabarro and Benjamin-Ono equations, J. Funct. Anal. 145 (1997), no. 1, 136-150. http://dx.doi.org/1 0.1006/jfan.1996.3016. MR1442163 (97m:35235)

[69] E. VALDINOCI, From the long jump random walk to the fractional Laplacian, Bol. Soc. Esp. Mat. Apl. SëMA 49 (2009), 33-44. MR2584076 (2011a:60174)

[70] E. VALDINOCI, B. SCIUNZI, and V.O. SAVIN, Flat level set regularity of p-Laplace phase transitions, Mem. Amer. Math. Soc. 182 (2006), no. 858, vi+144. MR2228294 (2007a:35050)

[71] G.B. Whitham, Linear and Nonlinear Waves, Wiley-Interscience [John Wiley \& Sons], New York, 1974. Pure and Applied Mathematics. MR0483954 (58 \#3905)

[72] V.E. ZAKHAROV, Stability of periodic waves of finite amplitude on the surface of a deep fluid, Zh. Prikl. Mekh. Tekh. Fiz. 9 (1968), 86-94.

ALBERTO FARINA:

LAMFA - CNRS UMR 6140

Université de Picardie Jules Verne

Faculté des Sciences

33, rue Saint-Leu, Amiens, France

E-MAIL: alberto.farina@u-picardie.fr

ENRICO VALDINOCI:

Università di Roma Tor Vergata

Dipartimento di Matematica

Rome, Italy

E-MAIL: enrico.valdinoci@uniroma2.it

KEY WORDS AND PHRASES: elliptic PDEs, fractional or nonlinear operators, symmetry results. 2000 Mathematics SubjeCt Classification: 35S05, 35J60, 35 J61.

Received: June 22, 2010; revised: September 9, 2010.

Article electronically published on September 9, 2010. 